\title{
LETTER \\ Speech-Act Classification Using a Convolutional Neural Network Based on POS Tag and Dependency-Relation Bigram Embedding
}

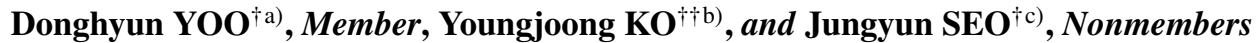

\begin{abstract}
SUMMARY In this paper, we propose a deep learning based model for classifying speech-acts using a convolutional neural network (CNN). The model uses some bigram features including parts-of-speech (POS) tags and dependency-relation bigrams, which represent syntactic structural information in utterances. Previous classification approaches using CNN have commonly exploited word embeddings using morpheme unigrams. However, the proposed model first extracts two different bigram features that well reflect the syntactic structure of utterances and then represents them as a vector representation using a word embedding technique. As a result, the proposed model using bigram embeddings achieves an accuracy of $89.05 \%$. Furthermore, the accuracy of this model is relatively $2.8 \%$ higher than that of competitive models in previous studies.

key words: speech-act classification, dependency-relation, convolutional neural network, word embedding, bigram embedding
\end{abstract}

\section{Introduction}

A speech-act is a linguistic action intended by a speaker, and speech-act classification refers to the analysis of the intention of the speaker in an utterance [1]. In particular, a speech-act classification must be highly accurate because it is the first step in processing an utterance in a dialogue system. However, it is difficult to perfectly interpret a speaker's intention because a speaker indicates his/her intention using various expressions [2]. In order to overcome this problem, most studies adopt supervised machine learning methods using various speech features including lexical, contextual, and other semantic features [3]-[5]. Recently, many deep learning techniques have been applied to natural language processing because they afford a significant advantage with regard to analyzing the semantics of lexical features based on a distributed representation. Thus, in this study, we attempt to improve the accuracy of speech-act classification through the use of better distributed representations for various features.

In this paper, we propose a new speech-act classification model using a convolutional neural network (CNN) based on bigram embedding. Most deep learning techniques use a distributed representation of features, called word embedding. Word embeddings are distributed representations

\footnotetext{
Manuscript received April 19, 2017.

Manuscript publicized August 23, 2017.

$\dagger$ The authors are with Sogang University, \#908 R-hall, Sinsudong, Mapo-gu, 121-742, Republic of Korea.

${ }^{\dagger}$ The author is with Dong-A University, 840, Hadan 2-dong, Saha-gu, Busan, 604-714, Republic of Korea.

a) E-mail: babuluve@gmail.com

b) E-mail: youngjoong.ko@gmail.com (Corresponding author)

c) E-mail: seoyj@ sogang.ac.kr

DOI: 10.1587/transinf.2017EDL8083
}

of words that group words that share semantic or syntactic properties. CNNs have traditionally used only morpheme unigram embeddings, whereas the proposed model additionally uses bigram embeddings created by various bigram features such as morpheme, parts-of-speech (POS) tags, and dependency-relation bigrams. In particular, a model with POS tags and dependency-relation bigrams achieves the highest accuracy because it can represent structural information of utterances.

The remainder of this paper is organized as follows. The next section summarizes related work. In Sect. 3, we explain our proposed method in detail. In Sect. 4, experimental results and comparisons between the proposed method and previous ones are presented. The paper is finally concluded in Sect. 5.

\section{Related Work}

Most previous studies for speech-act classification have used various supervised machine-learning methods to automatically identify a speaker's intention. Lee [3] designed a speech-act classification method using the hidden Markov model (HMM) to estimate speech-act probabilities and this method was improved upon by using smoothed class probabilities from a decision tree. Choi [4] proposed a maximum entropy model (MEM) to determine the speech-act of current utterances using previous utterances as contextual information. Song [5] recommended a support vector machine (SVM) model to preferentially analyze classes with lower distribution when training among a set of classes.

Since deep learning techniques have recently shown better performances in many fields such as image classification [6], many natural language researchers have applied deep learning techniques using word embeddings to many natural language processing problems [7], [8]. Collobert [7] constructed a model for various natural language processing techniques. Kim [8] presented a convolutional neural network model for sentence classification. These studies used word embeddings with a morpheme unit for the convolutional neural network, but we utilize bigram embeddings of various bigram features such as POS tag bigram and dependency-relation bigram, which contain syntactic structural information within utterances.

\section{Proposed Model}

Figure 1 outlines the proposed model, which consists of 


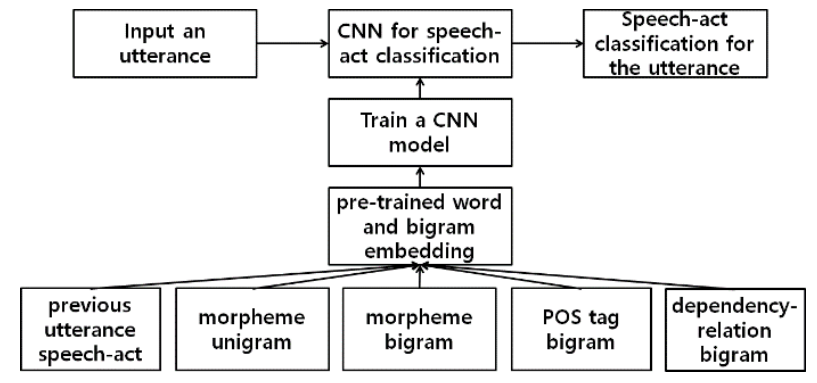

Fig. 1 Overview of speech-act classification using unigram and bigram embedding

three steps. The first step is the extraction of five types of features: previous utterance speech-act, morpheme unigram, morpheme bigram, POS tag bigram, and dependencyrelation bigram. In the next step, the extracted features are converted into a distributed representation using a word embedding technique during pre-training. Finally, $\mathrm{CNN}$ is trained with an effective combination of the extracted features.

\subsection{Word Embedding Technique}

Word embeddings express the latent values of features as distributed representations. They are trained using the neural network language model [9]. It is also possible to express words that are represented by large data in a real-valued vector form. Mikolov [10] proposed a neural network language model to remove the hidden layer for word embeddings: the continuous bag-of-words (CBOW) and Skip-gram model. CBOW predicts the current word based on the context, and Skip-gram specifies the word based on the context. In particular, the removal of the hidden layer results in faster training speed compared to other models.

\subsection{Convolutional Neural Network (CNN)}

A convolutional neural network (CNN) is a deep learning technique. In particular, many studies have proved the effectiveness of $\mathrm{CNN}$ for natural language processing. Kim proposed the CNN architecture for sentence classification in [8]. Here, we use Kim's CNN architecture for speech-act classification. However, unlike Kim's model, we applied bigram embeddings of various features into the $\mathrm{CNN}$ architecture to improve the performance of speech-act classification.

The CNN architecture has various layers. In the first layer, extracted features are transformed into a distributed presentation using pre-trained word embeddings. A convolution layer produces new features using a filter that applies to each window of words on a current word to produce a feature map. For the max pooling layer, the maximum value is selected from each feature map to capture the most important feature. The proposed model uses multiple filters to obtain multiple features. Finally, the max pooling layer is fully connected to the softmax layer whose output is the probability distribution over classes.

\subsection{Feature Extraction for Speech-Act Classification}

In previous studies, several kinds of features have been used for speech-act classification, such as lexical features (morpheme unigrams and POS tag bigrams) and a contextual feature (speech-act of previous utterance) [1], [11]. However, we believe that there exist more effective features, which can be used to further improve speech-act classification using feature distributed representations in deep learning. Thus, different features are considered in this research to reflect the syntactic information of utterances, such as morpheme and dependency-relation bigrams. Consequently, five types of features are used in speech-act classification: the speechact of previous utterance, morpheme unigram, morpheme bigram, POS tag bigram, and dependency-relation bigram.

In previous studies, the speech-act of previous utterances is considered important as the speech-act of the current utterance highly depends on that of the previous utterance as contextual information. In addition to the abovementioned lexical and contextual features, the proposed model attempts to utilize the syntactic information of utterances based on POS tags and dependency-relation bigrams for speech-act classification. In actual, a POS tag bigram provides a pattern of consecutive POS tags in the syntactic structure of an utterance and a dependency-relation bigram affords the same for syntactic information between constituents, such as subjects, objects, and verbs, in whole utterances; for example, consider the input utterance is "네, 이름을 알려주시고 얼마 동안 머무르실 건지 가르쳐 주십시오. (Yes, please tell me your name and how long you intend to stay here)", the analysis result from the dependency parser is shown in Fig. 2. They represent relationships between constituents and these relationships are called dependencyrelations. Choi [12]'s dependency parser is used in the paper. An arc represents relationships between two constituents analyzed by a dependency parser. Boxes in the first layer contain morphemes analyzed by a POS tagger and boxes in the second layer contain relationship names from the dependency parser.

However, we do not use all the head-dependent relationships in the result from the dependency parser. Relation (1) is excluded because it includes unnecessary syntactic information like interjections. Further, we use other relations to learn word embeddings. These head-dependent relationships are extracted as dependency-relation bigrams as shown in Figs. 2 and 3.

All the above-mentioned four types of features must be converted into vector representations to be used in CNN. These four features including morpheme unigram, morpheme bigram, POS tag bigram, and dependency-relation bigram are represented as vectors by a word embedding technique, whereas the speech-act of previous utterances is initialized randomly as the same size of the vector as that of the other three types of features.

Table 1 shows the four types of unigram and bigram features used in this research. Features are extracted through 


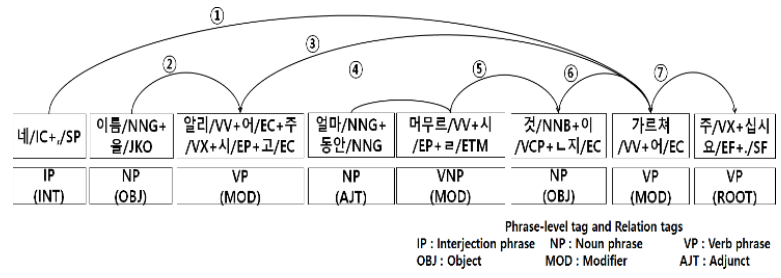

Fig. 2 Analysis result of a dependency parser

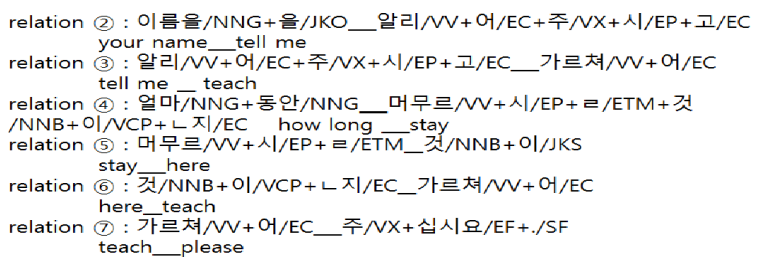

Fig. 3 Example of extracted dependency-relation bigrams

Table 1 Example of extracted bigram features

\begin{tabular}{|c|c|}
\hline Example & $\begin{array}{c}\text { 네, 이름을 알려주시고 얼마 동안 머무르실 건지 } \\
\text { 가르쳐 주십시오. (Yes, please tell me your name and } \\
\text { how long stay here) }\end{array}$ \\
\hline $\begin{array}{l}\text { Morpheme } \\
\text { unigram }\end{array}$ & 네/IC, 이름/NNG, 을/JKO, ... \\
\hline $\begin{array}{l}\text { Morpheme } \\
\text { bigram }\end{array}$ & $\begin{array}{c}\text { 네/IC_이름/NNG, 이름/NNG_을/JKO, } \\
\text { 을/JKO_알리 } / \mathrm{VV}, \ldots\end{array}$ \\
\hline $\begin{array}{c}\text { POS tag } \\
\text { bigram }\end{array}$ & IC_NNG, NNG_JKO, JKO_VV, ,.. \\
\hline $\begin{array}{l}\text { Dependency } \\
\text {-relation } \\
\text { bigram }\end{array}$ & $\begin{array}{l}\text { 이름을/NNG+을/JKO_알리 } / \mathrm{VV}+\text { 어/EC+주 } / \mathrm{VX}+\text { 시 } / \mathrm{EP}+\text { 고/EC, } \\
\text { 알리 } / \mathrm{VV}+\text { 어/EC+주 } / \mathrm{VX}+\text { 시/EP+고/EC_가르쳐 } / \mathrm{VV}+\text { 어/EC, }\end{array}$ \\
\hline
\end{tabular}

the following steps. First, morpheme unigram features are created by a lexical/POS_tag after a POS tagger analyzes the input utterance; morpheme bigrams are extracted using the sliding window technique with a window size of 2 . Then, the input utterance annotated by the POS tagger is syntactically analyzed by a dependency parser and the dependencyrelation bigrams are extracted by the processes explained in Figs. 2 and 3. Finally, the extracted unigram or bigram features are converted into distributed representations as vectors using the embedding technique of CBOW and they are used as inputs of the input layer of CNNs. The corpus for feature embedding is composed of articles from KBS News ${ }^{\dagger}$ and its size is approximately $3 \mathrm{G}$ bytes.

\section{Experiments}

In this section, we compare the proposed model with those proposed in previous studies over several experiments to demonstrate the effectiveness of the proposed model.

\subsection{Experimental Settings}

To evaluate the proposed model, we used the reservation

\footnotetext{
${ }^{\dagger}$ http://news.kbs.co.kr/
}

Table 2 Experiment results of combination of various unigram and bigram features

\begin{tabular}{|l|c|c|}
\hline & SVM & CNN \\
\hline $\begin{array}{l}\text { previous utterance speech-act } \\
+ \text { morpheme unigram }\end{array}$ & 85.05 & 88.23 \\
\hline $\begin{array}{l}\text { previous utterance speech-act } \\
+ \text { morpheme unigram } \\
+ \text { morpheme bigram }\end{array}$ & 84.65 & 87.29 \\
\hline $\begin{array}{l}\text { previous utterance speech-act } \\
+ \text { morpheme unigram } \\
+ \text { POS tag bigram }\end{array}$ & $\mathbf{8 5 . 9 5}$ & 88.35 \\
\hline $\begin{array}{l}\text { previous utterance speech-act } \\
+ \text { morpheme unigram } \\
+ \text { dependency-relation bigram }\end{array}$ & 85.89 & 88.11 \\
\hline $\begin{array}{l}\text { previous utterance speech-act } \\
+ \text { morpheme unigram } \\
+ \text { POS tag bigram } \\
\text { + dependency-relation bigram }\end{array}$ & 85.79 & $\mathbf{8 9 . 0 5}^{*}$ \\
\hline \\
* A significant test is conducted by one-tailed McNemar's test and the improvement of the \\
proposed CNN is statistically significant, $\mathrm{p}<0.01$ (p=0.000102).
\end{tabular}

tasks corpus that was used in the previous studies [3][5], [11] which was transcribed from real conversions occurring when making hotel, airline, and tour reservation. That corpus consists of 528 dialogues, 10,285 utterances, and 17 types of speech-acts. The corpus was divided into training (428 dialogues and 8,349 utterances) and testing data (100 dialogues and 1,936 utterances). Since the corpus has no standard development set, $20 \%$ of the training data was randomly selected as a development set. The development set is used for tuning the parameters of CNNs.

In order to evaluate speech-act classification, we used the accuracy measure in Eq. (1).

$$
\text { Accucary }=\frac{\text { Number of correct sentences }}{\text { Number of test sentences }}
$$

We exploited the architecture of Kim [8]'s model using rectified linear units (ReLU) as an activation function. The window sizes of filters are 3, 4, and 5 with 100 feature maps. Dropout rate is 0.5 , and mini-batch size is 50 with the Adadelta update rule [13]. The dimension of the embeddings is set to 64 .

\subsection{Experimental Results}

Table 2 shows the final experimental results for combinations of various features with SVM and CNN.

Overall, the performance of CNN is much better than that of SVM in all the combinations. For SVM, the best performance is the combination of morpheme unigram and POS tag bigram, but other SVMs with dependency-relation bigrams also achieve very similar performances. However, the best among CNNs is from the combination of morpheme unigram, POS tag bigram, and dependency-relation bigram. The relative difference of the best performance of SVM and $\mathrm{CNN}$ is $3.6 \%$.

The combination of morpheme unigram and the speech act of previous utterances is considered as a baseline model; its performance was fairly high. As three different bigrams are added to the baseline model, their effectiveness is evaluated through experiments. The highest accuracy 
Table 3 Comparisons with previous studies

\begin{tabular}{|l|c|}
\hline & Accuracy (\%) \\
\hline Lee's HMM [3] & 81.50 \\
\hline Choi' MEM [4] & 83.57 \\
\hline Kim's SVM [12] & 86.62 \\
\hline Song's SVM [5] & 86.54 \\
\hline Proposed model & $\mathbf{8 9 . 0 5}$ \\
\hline
\end{tabular}

was achieved when the baseline model was combined with POS tag and dependency-relation bigrams. The accuracy is $0.93 \%$ relatively higher than that of the baseline model. While dependency-relation bigrams contain syntactic structure information within the utterance, they do not contain information from the sequence of words. Thus, further improvements in the combination model can be realized by adding POS tag bigrams into dependency-relation bigrams.

\subsection{Comparison of the Proposed Model and Previous Studies}

As shown in Table 3, Kim's [11] model achieved the highest accuracy in previous models. However, our proposed model achieved $2.8 \%$ relatively higher performance than that of Kim's model.

\section{Conclusions}

In this study, we presented a CNN model for speech-act classification using POS tag and dependency-relation bigrams. The proposed CNN model using POS tag and dependency-relation bigrams obtained the highest accuracy of $89.05 \%$. We think that these features well reflect the syntactic information of utterances to speech-act classification.

In the future, we will continue to study how to reflect other information of utterances to realize higher performances in speech-act classification.

\section{Acknowledgements}

This work was supported by Institute for Information \& communications Technology Promotion (IITP) grant funded by the Korea government (MSIT) (No. 2017-0-00550, De- velopment of Automatic Text Data Labeling and Verification Tools for Machine Learning).

\section{References}

[1] S. Kim, Y. Lee, and J. Lee, "Korean Speech Act Tagging using Previous Sentence Features and Following Candidate Speech Acts," Journal of KISS: Software and Applications, vol.35, no.6, pp.374-385, 2008.

[2] C. Doran, J. Aberdeen, L. Damianos, and L. Hirschman, "Comparing Several Aspects of Human-Computer and Human-Human Dialogues," Current and New Directions in Discourse and Dialogue, vol.22, pp.133-159, 2003.

[3] S. Lee and J. Seo, "Korean Speech Act Analysis System Using Hidden Markov Model with Decision Trees," International Journal of Computer Processing of Oriental Languages, vol.15, no.3, pp.231-243, 2002.

[4] W.S. Choi, H. Kim, and J. Seo, "An Integrated Dialogue Analysis Model for Determining Speech Acts and Discourse Structures," IEICE Transactions on Information and Systems, vol.E88-D, no.1, pp.150-157, 2005.

[5] N. Song, K. Bae, and Y. Ko, "Effective Korean Speech-act Classification Using the Classification Priority Apllication and a Post correction Rules," Journal of KIISE, vol.43, no.1, pp.80-86, 2016.

[6] A. Krizhevsky, S. Ilya, and G.E. Hinton, "Imagenet classification with deep convolutional neural networks," Advances in neural information processing systems, vol.60, no.6, pp.84-90, 2017.

[7] R. Collobert, J. Weston, L. Bottou, M. Karlen, K. Kavukcuoglu, and P. Kuksa, "Natural language processing (almost) from scratch," The Journal of Machine Learning Research, vol.12, pp.2493-2537, 2011.

[8] Y. Kim, "Convolutional neural networks for sentence classification," In Proceedings of EMNLP, pp.1746-1751, 2014.

[9] Y. Bengio, R. Ducharme, P. Vincent, and C. Jauvin, "A neural probabilistic language model," Journal of Machine Learning Research, pp.1137-1155, 2003.

[10] T. Mikolov, W.T. Yih, and G. Zweig, "Linguistic Regularities in Continuous Space Word Representations," InHLT-NAACL, vol.13, pp.746-751, 2013.

[11] K. Kim and J. Seo, "Decision of the Korean Speech Act using Feature Selection Method," Journal of KISS: Software and Applications, vol.30, no.3.4, pp.278-284, 2003.

[12] D. Choi, J. Park, and K. Choi, "Korean treebank transformation for parser training," Proceedings of the ACL 2012 Joint Workshop on Statistical Parsing and Semantic Processing of Morphologically Rich Languages, vol.29, no.6, pp.78-88, 2012.

[13] M.D. Zeiler, "ADADELTA: An adaptive learning rate method," arXiv preprint arXiv:1212.5701, 2012. 